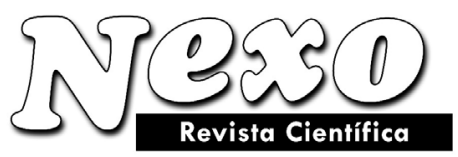

Vol. 23, No. 01, pp.18-26/Mayo 2010

\title{
Estimación de la carga de nutrientes procedentes de la cuenca de drenaje superficial del rio Tepenaguasapa
}

\author{
B. Morales ${ }^{1 *}$, G. Chávez ${ }^{2}$ \\ ${ }^{1}$ Ministerio del Ambiente y los Recursos Naturales (MARENA) \\ Km 12 1/2 Carretera Norte, Managua, Nicaragua \\ e-mail: bismarckmorales@yahoo.com \\ ${ }^{2}$ CEMEX de Nicaragua \\ PO Box 75, Managua, Nicaragua \\ e-mail: gachaly@hotmail.com
}

(recibido/received: 22-Marzo-2010; aceptado/accepted: 04-Mayo-2010)

\begin{abstract}
RESUMEN
El Lago Cocibolca merece atención especial por ser una fuente importante de agua para el consumo humano. El río Tepenaguasapa es una de sus subcuencas, que tiene entre otros problemas, la contaminación con nutrientes. Para el estudio de este problema, fue necesario determinar la carga de nutrientes (Fósforo y Nitrógeno) procedentes de las subcuencas. La carga de Fósforo estimada fue de 24.33 t/año y de Nitrógeno de 632.57 t/año, para totalizar 656.89 t/año, con un orden de importancia por su origen de: uso de suelo, precipitación y escurrimiento artificial. El río varía en su estado trófico con un gradiente de concentración de nutrientes aguas arriba hacia aguas abajo. La carga de nutrientes es originada por la gran cantidad de área empleada para pasto y actividad ganadera extensiva, tierras de labranza, monocultivo, manejo de las aguas residuales, tala de árboles, además de ser una cuenca con abundante precipitación y de gran tamaño en comparación con el espejo de agua.
\end{abstract}

Palabras clave: fósforo, nitrógeno, actividad ganadera extensiva, carga de nutrientes, estado trófico.

\begin{abstract}
Lake Cocibolca merits special attention as an important source of potable water. The Tepenaguasapa River has a sub watertable which, among other problems, has nutrient contamination. For research into this problem it was necessary to determinate the amount of nutrients (Phosphorus and Nitrogen) in the watertable. The amount of Phosphorus estimated was $24.33 \mathrm{t} /$ year and Nitrogen $632.57 \mathrm{ft} /$ year making a total of $656.89 \mathrm{t} /$ year with an order of importance in regard to origin, use of soil, rainfall and artificial water runoff. The river varies in it's trophic state with a concentration of nutrients in a gradient from upstream to downstream. The nutrient load originates, for the most part, in areas used for pasture and livestock, farming monoculture, waste water management and logging, as well as a river basin which has an abundant rainfall and is of large size compared to the surface water.
\end{abstract}

Keywords: phosphorus, nitrogen, extensive livestock activities, nutrient loading, trophic state.

${ }^{*}$ Autor para la correspondencia 


\section{INTRODUCCIÓN}

El Fósforo (P) y Nitrógeno $(\mathrm{N})$ son considerados los principales nutrientes en el desarrollo de los ecosistemas acuáticos. En grandes cantidades estos nutrientes originan el problema de la eutrofización de los cuerpos de agua, lo que es provocado ya sea de forma natural o antropogénico.

Las acciones antropogénicos más importantes sobre los bosques de la Cuenca del Río San Juan son: a) destrucción del hábitat por la extracción forestal con fines comerciales, y b) el avance de la frontera agrícola y ganadera, los cuales originan la contaminación y sedimentación de los cuerpos de agua. Estos últimos son consecuencia de la deforestación, la agricultura, la ganadería, y la inadecuada disposición de los desechos sólidos y líquidos provenientes de los asentamientos humanos y de la actividad industrial (Romero, J. et al, 2004).

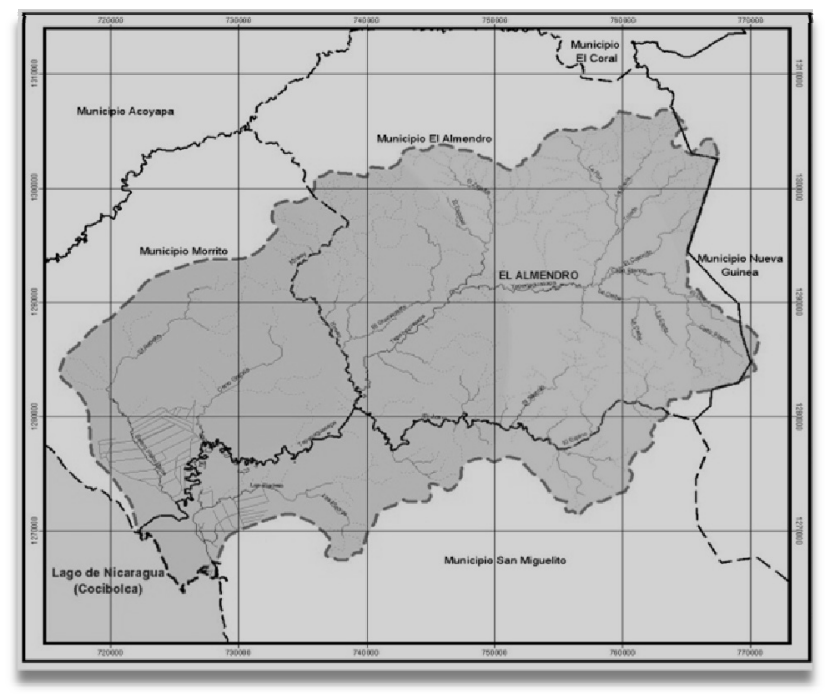

Figura 1. Mapa de la cuenca del Río Tepenaguasapa.

Como resultado, surge el problema de eutrofización cultural. Uno de los cuerpos de agua que pueden ser afectados por este fenómeno es el Lago Cocibolca, una reserva de agua de mucha importancia para Nicaragua. Alrededor de este lago, existen una serie de subcuencas que igualmente pueden ser afectadas, y por tanto, contribuir al deterioro del Lago Cocibolca.

Entre estas subcuencas se encuentra la del Río Tepenaguasapa (ver figura 1) que en la actualidad, y dadas sus características ambientales está generando un aporte significativo de nutrientes de Fósforo (P) y Nitrógeno (N) al lago.
Para estimar el exceso de carga de nutrientes y la consecuente eutrofización en el ecosistema, en la cuenca de drenaje superficial del Río Tepenaguasapa, es necesario conocer los valores de carga de nutrientes con metodologías confiables.

En los últimos años, han sido desarrollados métodos para la realización de análisis empíricos y teóricos, destinados a estimar la eutrofización de lagos, de forma tal, que estos puedan ser fácilmente aplicables. Estos esfuerzos han conseguido obtener como resultado, el desarrollo de una metodología para la estimación de la carga de nutrientes en lagos, y un modelo para la evaluación de eutrofización en lagos cálidos tropicales.

El método cálculo de la carga de nutrientes en lagos ha sido aplicado para estimar la cantidad de Fósforo y Nitrógeno escurridos en la cuenca de drenaje superficial del Río Tepenaguasapa, y el estado trófico de sus aguas fue valorado por medio del método: Modelo Simplificado para la Evaluación de Eutrofización en Lagos Cálidos Tropicales.

Una vez conocidos estos parámetros, y por la importancia que implica la conservación de las subcuencas que drenan agua hacia el Lago Cocibolca, (caso del Río Tepenaguasapa), se contó con información que permitió conocer la cantidad de escurrimiento de los nutrientes Fósforo $(\mathrm{P})$ y Nitrógeno $(\mathrm{N})$, y su relación con las actividades antropogénicas. Asimismo, se formularon propuestas para la solución a los problemas presentados, así como acciones y medidas para la mejora de la calidad del agua, su protección y la de todos los habitantes la cuenca. De esta forma, se aportó a la conservación de esta fuente de agua, considerada como la de mayor importancia para el futuro inmediato de Nicaragua.

\section{METODOLOGÍA}

Los modelos empleados en la cuenca hidrográfica, son modelos simples y confiables que son aplicados en los casos de no contar con la suficiente cantidad de información ni de disponer de grandes recursos económicos (caso de Nicaragua). Sin embargo, debe tenerse en consideración, que el modelo desarrollado posee un mayor grado de confiabilidad entre más se aproximen sus datos a la realidad (dada la cantidad de factores que este toma en cuenta), esto posibilita la 
obtención de una mejor caracterización de lo que ocurre en la cuenca.

\section{Método Cálculo de Carga de Nutrientes}

La metodología empleada para estimar el escurrimiento de Fósforo y Nitrógeno de la Cuenca del Río Tepenaguasapa es conocida como: Cálculo de la Carga de Nutrientes en Lagos (JØrgensen, S., Vollenweider, R. 1988), y consiste en la aplicación de una serie de fórmulas que permiten calcular la carga de Fósforo y Nitrógeno provenientes de las variables: precipitación en forma de lluvia, escurrimiento natural del suelo y por carga artificial.

- Estimación de Fósforo y Nitrógeno por Precipitación

Para el cálculo de este dato, se utilizó la información oficial obtenida del Sistema Nacional de Información Ambiental (SINIA), quienes elaboraron los mapas sobre esta variable, así como los del resto de variables.

La cuenca del Río Tepenaguasapa, es relativamente grande (133 $\left.435.36 \mathrm{ha} ; 1334.35 \mathrm{~km}^{2}\right)$, además se encuentra dentro de un gran rango de precipitación media anual, que va desde los $2400 \mathrm{~mm}$ anuales hacia el este de la cuenca (zona alta), hasta los $1400 \mathrm{~mm}$ anuales (zona baja) en dirección oeste de la cuenca, lo que genera un rango de diferencia de $1000 \mathrm{~mm}$.

Debido a su amplio rango e intensidad, se tomó la decisión de subdividir la precipitación en cuatro regiones de menor a mayor, con intèrvalos de $200 \mathrm{~mm}$ en $200 \mathrm{~mm}(1,400$ a 1,$600 ; 1,600$ a 1,$800 ; 1,800$ a 2,000$)$ hasta llegar al sector que se encuentra más arriba en la cuenca, asignándole un rango de $400 \mathrm{~mm}(2,000$ a 2,400 $\mathrm{mm}$ anuales), esto debido a la poca área que esto representa y que esta precipitación no cae directamente sobre el Río Tepenaguasapa.

La cuenca está ubicada dentro de dos zonas ecológicas (Salas, 2002), las cuales tienen características particulares, ya que la parte baja pertenece a la Región Ecológica III (Central Sur), que a su vez, por la diversidad en sus características de vegetación y ecología, está subdivida en cinco subregiones.

Los valores medios de concentraciones $\left(\mathrm{C}_{\mathrm{PP}}\right.$ y $\left.\mathrm{C}_{\mathrm{NP}}\right)$ utilizados para el Fósforo (P) y Nitrógeno $(\mathrm{N})$, en la precipitación se pueden apreciar en el Tabla 1.
Tabla 1 Valor medio de concentraciones de $\mathrm{P}$ y N.

\begin{tabular}{|c|c|c|}
\hline Precipitación & $\mathbf{C}_{\mathbf{P P}}$ & $\mathbf{C}_{\mathbf{N P}}$ \\
\hline Rango & $0.025-0.1$ & $0.3-1.6$ \\
\hline Media & 0.07 & 1.0 \\
\hline
\end{tabular}

Fuente: (Jørgensen, S., Vollenweider, R. 1988)

Las estimaciones de nutrientes por precipitación están dadas por las fórmulas 1 y 2 :

$$
\begin{aligned}
\mathrm{I}_{\mathrm{PP}} & =\mathrm{PC}_{\mathrm{PP}} \times \mathrm{A}_{\mathrm{S}} \\
\mathrm{I}_{\mathrm{NP}} & =\mathrm{PC}_{\mathrm{NP}} \times \mathrm{A}_{\mathrm{S}}
\end{aligned}
$$

donde

$\mathrm{A}_{\mathrm{S}}=\quad$ Área superficial del Cuerpo de Agua en metros cuadrados $\left(\mathrm{m}^{2}\right)$

$\mathrm{C}_{\mathrm{PP}}=$ Concentración de Fósforo por Precipitación

$\mathrm{C}_{\mathrm{NP}}=$ Concentración de Nitrógeno por Precipitación

$\mathrm{P}=\quad$ Precipitación Media Anual de la Cuenca

$I_{P P}=$ Carga de Fósforo por Precipitación

$\mathrm{I}_{\mathrm{NP}}=$ Carga de Nitrógeno por Precipitación

- Estimación de Fósforo y Nitrógeno por Uso de Suelo

Para calcular esta variable, se clasificó el escurrimiento según el origen en:

1) bosque

2) bosque+pastura

3) áreas agrícolas

Los valores promedios de exportación de Fósforo $\left(\mathrm{E}_{\mathrm{P}}\right) \mathrm{y}$ Nitrógeno $\left(\mathrm{E}_{\mathrm{N}}\right)$ empleados se pueden apreciar en la Tabla 2.

Antes de la aplicación de estos promedios, fue necesario caracterizar las formaciones geológicas presentes en la cuenca y adecuarlas al método. Posteriormente, fueron aplicadas las fórmulas 3 y 4 :

$$
\begin{aligned}
& \mathrm{I}_{\mathrm{Pt}}\left(\mathrm{mg} \mathrm{a}^{-1}\right)=\Sigma_{\mathrm{i}=1} \mathrm{~A}_{\mathrm{t}}\left(\mathrm{m}^{2}\right) \times \mathrm{E}_{\mathrm{P}}\left(\mathrm{mg} \mathrm{m}^{-2} \mathrm{a}^{-1}\right) \\
& \mathrm{I}_{\mathrm{Nt}}\left(\mathrm{mg} \mathrm{a}^{-1}\right)=\Sigma_{\mathrm{i}=1} \mathrm{~A}_{\mathrm{t}}\left(\mathrm{m}^{2}\right) \times \mathrm{E}_{\mathrm{N}}\left(\mathrm{mg} \mathrm{m}^{-2} \mathrm{a}^{-1}\right)
\end{aligned}
$$

donde

E $\left(m g \mathrm{~m}^{-2} \mathrm{a}^{-1}\right)=$ Carga Natural de Fósforo (miligramos $/ \mathrm{m}^{2} *$ año).

$\mathrm{E}_{\mathrm{N}}\left(\mathrm{mg} \mathrm{m} \mathrm{m}^{-2} \mathrm{a}^{-1}\right)=$ Carga Natural de Nitrógeno (miligramos $/ \mathrm{m}^{2} *$ año)

$\mathrm{A}_{\mathrm{t}}=$ Área total $\left(\mathrm{m}^{2}\right)$

$\mathrm{I}_{\mathrm{Pt}}=$ Exportación de fósforo (miligramos/año)

$\mathrm{I}_{\mathrm{Nt}}=$ Exportación de nitrógeno (miligramos/año) 
Tabla 2 Valores de Exportación de Fósforo $\left(E_{\mathrm{P}}\right)$ y Nitrógeno $\left(\mathrm{E}_{\mathrm{N}}\right)$.

\begin{tabular}{|c|c|c|c|c|}
\hline \multirow{2}{*}{ Uso del suelo } & \multicolumn{2}{|c|}{ Ep $\left[\mathrm{mg} \cdot \mathrm{m}^{-2} \mathrm{año}^{-1}\right]$} & \multicolumn{2}{|c|}{ En $\left[\mathrm{mg} \cdot \mathrm{m}^{-2} \mathrm{año}^{-1}\right]$} \\
\hline & Ígnea & Sedimentaria & Ígnea & Sedimentaria \\
\hline $\begin{array}{l}\text { Escurrimiento } \\
\text { en Bosque }\end{array}$ & $0.7-9$ & $7-18$ & $\begin{array}{l}130- \\
300\end{array}$ & $150-500$ \\
\hline Rango Media & 4.7 & 11.7 & 200 & 340 \\
\hline $\begin{array}{l}\text { Bosque } \\
\text { Pastura }\end{array}$ & $6-12$ & $11-37$ & $\begin{array}{l}200 \\
600\end{array}-$ & $300-800$ \\
\hline Rango Medio & 10.2 & 23.3 & 400 & 600 \\
\hline $\begin{array}{l}\text { Áreas } \\
\text { Agrícolas }\end{array}$ & & & & \\
\hline Cítricos & 18 & & & 2240 \\
\hline Pastura & $15-75$ & & & $100-850$ \\
\hline Labranza & $22-100$ & & & $500-1200$ \\
\hline
\end{tabular}

(Jørgensen, S. Vollenweider, R. 1988)

- Estimación de Fósforo y Nitrógeno por Carga Artificial

La estimación de la población humana existente en la cuenca fue calculada utilizando los datos de los censos poblacionales llevados a cabo en los años 1995 (INEC, 1995), y 2005 (INIDE, 2007).

Se tomaron las cifras de las poblaciones de los municipios de Morrito, San Miguelito, el Almendro y Nueva Guinea. Posteriormente se empleó la fórmula 5, para estimar la población actual dentro de cada municipio:

donde

$$
\mathrm{R}=\left(\mathrm{Pd} / \mathrm{P}_{0}\right)^{1 / \mathrm{n}}-1
$$

$\mathrm{R}=$ Razón de Crecimiento [ ]

$\mathrm{P}_{0}=$ Población de cada municipio en el año base cero (1995).

Pd $(2005)=$ Población de cada municipio en el año de interés (2005).

$\mathrm{n}=$ Número de años transcurridos desde 0 hasta $\mathrm{d}$.

Una vez calculado el valor $\mathrm{R}$, se realizó una proyección para obtener un estimado de la población de habitantes $\left(\mathrm{P}_{2008}\right)$ en los cuatro municipios de interés. Para la obtención de este valor se empleó la fórmula 6 .

$$
\mathrm{Pd}=\mathrm{P} 0 *(1+\mathrm{R})^{\mathrm{n}}
$$

donde
Pd $=$ Población de cada municipio en el año de interés (2008).

$\mathrm{P}_{0}=$ Población de cada municipio en el año base cero (2005).

$\mathrm{R}=$ Razón de Crecimiento [ ]

$\mathrm{n}=$ Número de años transcurridos desde 0 hasta $\mathrm{d}$.

Se tomaron los valores de referencia suministrados por el método. Estos pueden ser apreciados en la Tabla 3:

Tabla 3 Medias de las Descargas Per- Cápita Anuales de Fósforo y Nitrógeno.

\begin{tabular}{|c|c|c|}
\hline Nutrientes & Descargas per-cápita anuales & Media \\
\hline Fósforo & $800-1800 \mathrm{~g}$ & 1,200 \\
\hline Nitrógeno & $300-3800 \mathrm{~g}$ & 3,400 \\
\hline
\end{tabular}

Fuente: (JØrgensen, S. Vollenweider, R. 1988)

Finalmente, para obtener un estimado de la cantidad de población, se tomaron los datos del área de cada municipio perteneciente a la cuenca del Río Tepenaguasapa, y se hizo una operación de relación entre el área y la proyección de la población, representada en las fórmulas 7 y 8 :

donde:

$$
\begin{gathered}
\mathrm{I}_{\mathrm{Pw}}=\mathrm{E}_{\mathrm{P}} \times \Sigma \mathrm{ha} \\
\mathrm{C}_{\mathrm{Nw}}=\mathrm{E}_{\mathrm{N}} \times \Sigma \mathrm{ha}
\end{gathered}
$$

$\mathrm{I}_{\mathrm{Pw}} \quad=$ Carga Artificial de Fósforo

$\mathrm{I}_{\mathrm{Nw}} \quad=$ Carga Artificial de de Nitrógeno

$\mathrm{E}_{\mathrm{P}} \quad=$ Carga Anual Per cápita de Fósforo

$\mathrm{E}_{\mathrm{N}} \quad=$ Carga Anual Per cápita de Nitrógeno

$\Sigma$ ha $\quad=$ Total de habitantes de la cuenca

Carga total de fósforo y nitrógeno (nutrientes) escurrida hacia el Río Tepenaguasapa.

La carga total de Fósforo y Nitrógeno se obtuvo de la sumatoria de los resultados de las cargas provenientes por precipitación, uso de suelo y cargas artificiales de Nitrógeno y Fósforo. Está sumatoria es reflejada en las fórmulas 9 y 10 :

$$
\begin{gathered}
\mathrm{I}_{\mathrm{P}}=\mathrm{I}_{\mathrm{PP}}+\mathrm{I}_{\mathrm{Pt}}+\mathrm{I}_{\mathrm{Pw}} \\
\mathrm{I}_{\mathrm{N}}=\mathrm{I}_{\mathrm{NP}}+\mathrm{I}_{\mathrm{Nt}}+\mathrm{I}_{\mathrm{Nw}}
\end{gathered}
$$

Los valores de estas variables fueron sumadas y permitieron conocer la Carga Total de Nutrientes (CTN) que son depositadas en el Río Tepenaguasapa anualmente. Lo anterior es denotado por la fórmula 11: 


$$
\mathrm{CTN}=\mathrm{I}_{\mathrm{P}}+\mathrm{I}_{\mathrm{N}}
$$

Metodología Simplificada para la Evaluación de Eutrofización en Lagos Cálidos Tropicales

Para la estimación del estado trófico del Río Tepenaguasapa se utilizó el Modelo Simplificado para la Evaluación de Eutrofización en Lagos Cálidos Tropicales (Salas, et al. 2001).

El desarrollo de la metodología simplificada para la evaluación de eutrofización en lagos cálidos tropicales (modelo de fósforo total, desarrollado por el CEPIS/HPE/OPS) utiliza 39 datos obtenidos de 27 lagos/embalses de América Latina y el Caribe. Con esta información, es posible elaborar un análisis de forma apropiada. Estos datos incluyen una amplia diversidad de condiciones limnológicas que van desde Oligotrófico a Hipereutrófico, clasificación trófica, y de profundidades en lagos que van desde someras a muy profundos y donde en la mayoría de los lagos está limitada por el fósforo.

Las figuras 1, 2 y 3 presentan en forma de nomogramas la distribución probabilística en el que se ubicaron los niveles de concentración total de fósforo encontradas en el Río Tepenaguasapa.

Para establecer el estado trófico del Río Tepenaguasapa, fueron tomadas muestras en las partes alta, media y baja del río. Las muestras fueron analizadas por el Laboratorio de aguas residuales del Centro de Investigación y Estudios en Medio Ambiente (CIEMA).

\section{RESULTADOS Y DISCUSIÓN}

Los resultados obtenidos del cálculo de carga de nutrientes, son resumidos numéricamente en la Tabla 4. En ella se aprecia, que el total de Fósforo y Nitrógeno vertido al Río Tepenaguasapa es de 656.89 t/año. De este total, 24.33 t/año corresponden a Fósforo y 632.57 t/año a Nitrógeno. El porcentaje que representan estas cantidades son de 3.70 y 96.30 respectivamente.

Las cantidades de Fósforo por uso de suelo resultaron ser las de mayor aportación, con 23.52 t/año (96.69\%), seguido por las procedentes de precipitación con 0.78 t/año $(3.20 \%)$ y por último las aportaciones por fuente artificial con 0.02 t/año, que representaron el $0.1 \%$. En el caso del Nitrógeno, y al igual que en el Fósforo un total de 621.35 t/año fueron aportadas por el uso del suelo (98.23\%), seguido por las precipitación con 11.15 t/año $(1.76 \%)$ y para la fuente artificial el Nitrógeno resultó ser de 0.07 t/año para el $0.01 \%$.

En lo que respecta a la relación de Fósforo y Nitrógeno (Tabla 4): la exportación por precipitación, dio como resultado $6.54 \%$ para el Fósforo, y $93.46 \%$ para el Nitrógeno. Para el caso de la exportación por uso de suelo, el Fósforo resultó en $3.65 \%$, y para el Nitrógeno en $96.35 \%$. Finalmente, para la exportación por fuente artificial el Fósforo resultó en $26.15 \%$ y el Nitrógeno en $73.85 \%$.

Con relación al total (la fuente de emisión, y el nutriente más representativo) y muy por encima de las demás, resultó ser el Nitrógeno procedente del suelo con 621.35 t/año, que significa el $94.59 \%$. En cuanto al Fósforo, de forma similar que el anterior, la fuente de mayor emisión fue el uso de suelo, con 23.52 t/año, con un $3.58 \%$ con respecto al gran total (ver Tabla 4 )

Tabla 4 Carga de $\mathrm{P}$ y $\mathrm{N}$ provenientes del drenaje superficial.

\begin{tabular}{|c|c|c|c|c|c|c|c|c|c|c|}
\hline \multirow{2}{*}{ CARGAS } & \multirow{2}{*}{$\begin{array}{c}\mathrm{P} \\
\text { Ion/Año }\end{array}$} & \multicolumn{3}{|c|}{ PORCENT AJE P } & \multirow{2}{*}{ N Ton/Año } & \multicolumn{3}{|c|}{ PORCENTAJE N } & \multirow{2}{*}{$\begin{array}{l}\text { CARGA } \\
\text { TOTAL } \\
\text { Ton/ } \\
\text { Año }\end{array}$} & \multirow{2}{*}{$\begin{array}{c}\% \\
\text { TOTAL }\end{array}$} \\
\hline & & P/P & $\mathrm{P} / \mathrm{N}$ & TOTAL & & $\mathrm{N} / \mathrm{N}$ & $\mathrm{PN}$ & TOTAL & & \\
\hline $\begin{array}{l}\text { Precipitación } \\
\text { Atmosférica. }\end{array}$ & 0.781 & 3.21 & 6.54 & 0.12 & 11.156 & 1.76 & 93.46 & 1.70 & 11.94 & 1.82 \\
\hline $\begin{array}{l}\text { Procedentes } \\
\text { del suelo. }\end{array}$ & 23.52 & 96.69 & 3.65 & 3.58 & 621.35 & 98.23 & 9635 & 94.59 & 644.87 & 98.17 \\
\hline Artificales & 0.02 & 0.10 & 26.15 & 0.004 & 0.07 & 0.01 & 73.85 & 0.01 & 0.09 & 0.01 \\
\hline Gran Total & 24.33 & 100.00 & & 3.70 & 632.57 & 100.00 & & 96.30 & 656.89 & 100.00 \\
\hline
\end{tabular}

En cuanto a la definición de cuál fue la fuente más representativa con respecto a la carga total (Tabla 4), se encontró que fue la procedente del suelo con 644.87 t/año $(98.17 \%)$, seguida por la precipitación atmosférica con 11.94 t/año $(1.82 \%)$ y por último se ubico la fuente artificial con 0.09 t/año $(0.01 \%)$.

De lo anterior queda establecido que el uso del suelo es la fuente de emisión que más contribuye al escurrimiento del Fósforo y Nitrógeno en la cuenca del Río Tepenaguasapa.

Cabe destacar que dentro de esta variable, el aporte proveniente de la combinación de escurrimiento en pastura, y bosque mas pastura fueron las más representativas, con un total de $1202,201460 \mathrm{~m}^{2}$, que 
equivalen al $90.1 \%$ del área total de la cuenca, lo cual se vincula con el alto grado de intervención humana que tiene la zona por las diversas actividades, sobre todo la que tiene que ver con la ganadería extensiva.

Contrario a lo anterior, se encontró que las fuentes de emisión, tanto artificial como por precipitación, fueron las de menor valor. Por el contrario, el uso del suelo de la cuenca genera un importante arrastre de sedimentos y en consecuencia, ocasiona la pérdida de nutrientes del mismo, esta situación es provocada por el excesivo uso del suelo para la actividad ganadera extensiva.

Por otro lado, la precipitación en la cuenca del Río Tepenaguasapa, es alta, (de $1400 \mathrm{~mm}$ a $2400 \mathrm{~mm}$ anuales), por lo que se infiere que representa un buen aporte de nutrientes por esta vía, aunque el espejo de agua sea de dimensiones modestas. Además, se debe agregar, que el Nitrógeno se mueve más dentro del medio ambiente que el Fósforo, por lo que consecuentemente habrá un mayor aporte de este último por esta fuente.

De igual forma, se debe consideró que la cuenca esta ubicada en una zona de transición que va desde un tipo de ecosistema de mayor precipitación hacia otro de modesta precipitación (Salas, 2002).

Por otro lado, la cuenca del Río Tepenaguasapa tiene $873,124440 \mathrm{~m}^{2}$ de formaciones geológicas de origen sedimentario, y 461,229180 $\mathrm{m}^{2}$ de origen ígneo. Esto es un dato importante, pues el método establece valores en dependencia de estas variables (Tabla 2), y como resultado, debido a que la mayoría del área es de formación sedimentaria, con el uso de la ganadería extensiva y la escasa vegetación, habrá por tanto un gran aporte de nutrientes.

La población de la cuenca resultó en 19498 habitantes, lo que permitió estimar una densidad poblacional aproximada de 15 habs. $/ \mathrm{km}^{2}$. Esto implica en términos poblacionales representa, pocos habitantes, y, dadas las características de ser una cuenca relativamente grande, existe una conjugación para que el aporte por esta fuente sea mínima, de hecho, estas aportaciones se podrían reducir aun mas, si se ejecutasen programas de saneamiento rural, ya que se encontró que en la actualidad no existen sistemas de tratamiento de aguas residuales, solamente letrinas, e incluso se practica el fecalismo al aire libre.
La cantidad de nutrientes que aporta el Río Tepenaguasapa al Lago Cocibolca es considerable. Se trata de uno de los mayores afluentes (con mayor caudal) del sector noreste de la cuenca, por lo que es indispensable que el gobierno central efectué un proceso de intervención para disminuir dichos aportes, y, de esa manera conserve la fuente de agua de mayor importancia en el futuro del país.

Por otra parte, los incendios forestales y puntos de calor detectados en Nicaragua deben ser considerados, puesto que se trata de dos factores que no se encuentran incorporados dentro del modelo aplicado y que en Nicaragua constituyen una variable a ser tomada en cuenta, debido a que estos provocan la pérdida de la biomasa, (principalmente vegetal), y la desnudez del suelo, que facilita a su vez el arrastre de los nutrientes por efecto de las condiciones climáticas, sean estas producidas por medio del viento o por precipitación. Adicionalmente se debe considerar que la cuenca está dominada por amplias zonas con pastizales los cuales tienden a quemarse durante la época seca.

Como resultado de lo antes señalado, las cenizas generadas por los incendios contienen Fósforo y Nitrógeno, que en su momento constituyeron parte de la masa vegetal y animal, las que posteriormente son transportadas hacia la cuenca y a las fuentes de agua.

\section{Estimación del Estado Trófico del Ecosistema}

Las concentraciones encontradas a partir de los analisis fueron de: $117.67 \mathrm{mg} / \mathrm{m}^{3}$ en la parte alta, $108.33 \mathrm{mg} / \mathrm{m}^{3}$ en la parte media y de $173.33 \mathrm{mg} / \mathrm{m}^{3}$ en la parte baja.

En las Figuras 2, 3 y 4 se observan las distribuciones de probabilidad para los puntos de muestreo de la parte alta, media y baja respectivamente. El resultado obtenido es la existencia de un estado mayormente eutrófico $(70 \%)$ para el punto de muestreo ubicado en la parte alta de la cuenca, para luego obtener un $17 \%$ de probabilidad de Hipertrófico, y de un $13 \%$ de probabilidad para mesotrófico.

En la parte media, el comportamiento es similar, con $72 \%$ de probabilidad de eutrófico, $16 \%$ para mesotrófico y $12 \%$ para hipertrófico.

Finalmente en la parte baja la probabilidad de eutrófico disminuye hasta el $58 \%$, sin embargo la probabilidad de hipertrófico aumenta hasta el 34\% y la probabilidad de mesotrófico decae hasta un $8 \%$. 
Estos resultados evidencian un comportamiento longitudinal de la carga de nutrientes durante el trayecto del cuerpo de agua. Es decir, entre más se aproxima el curso de agua hacia su desembocadura, existe una mayor concentración de nutrientes.

En este cuerpo de agua lótico se pueden dar cambios en las características del agua de una manera más fácil que en un cuerpo de agua léntico, arrastrando material, sobre todo en las crecidas, las cuales ocurren durante el período lluvioso. Este transporte de material aumenta con el uso del suelo lo cual es característico de la cuenca.

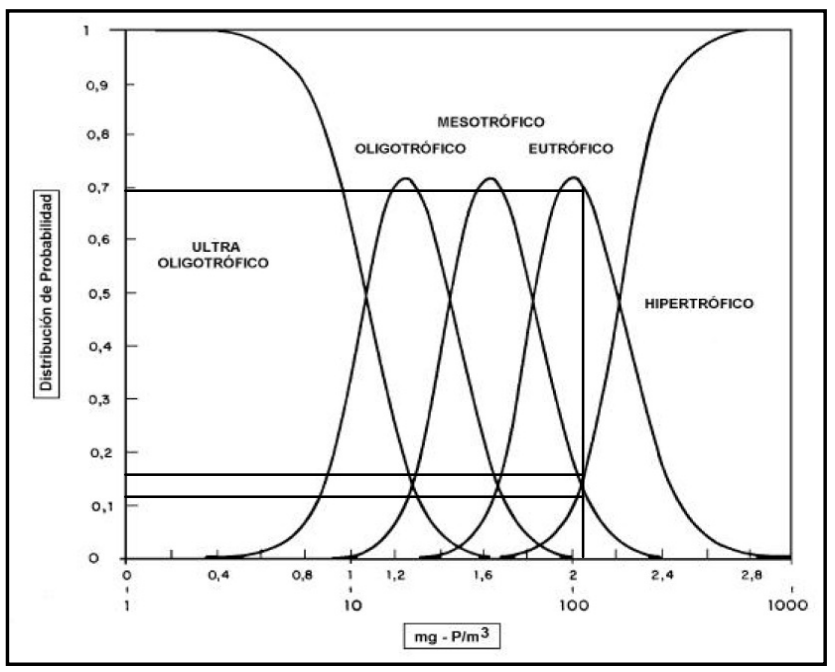

Fig. 2 Distribución de Probabilidad Estado Trófico Parte Alta Río Tepenaguasapa

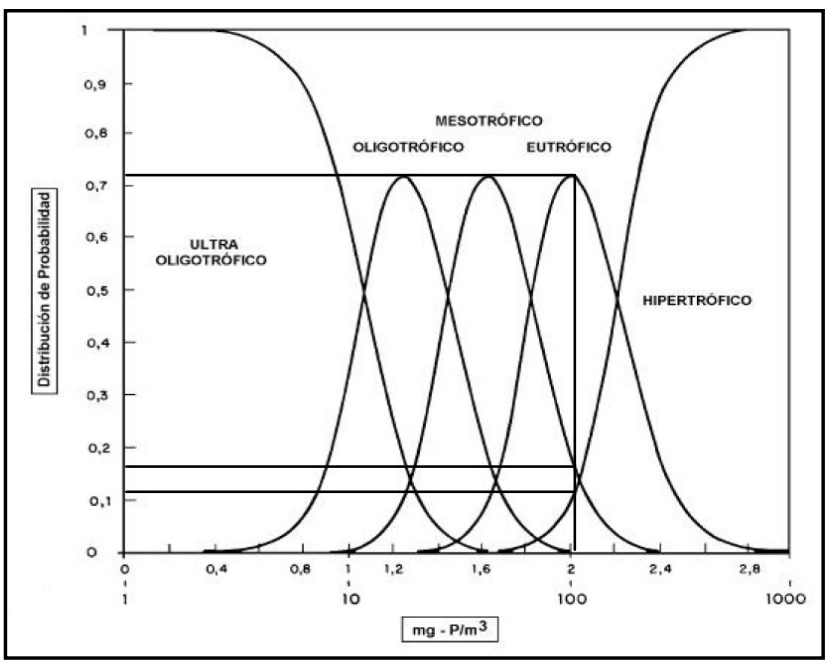

Fig. 3 Distribución de Probabilidad Estado Trófico Parte Media Río Tepenaguasapa

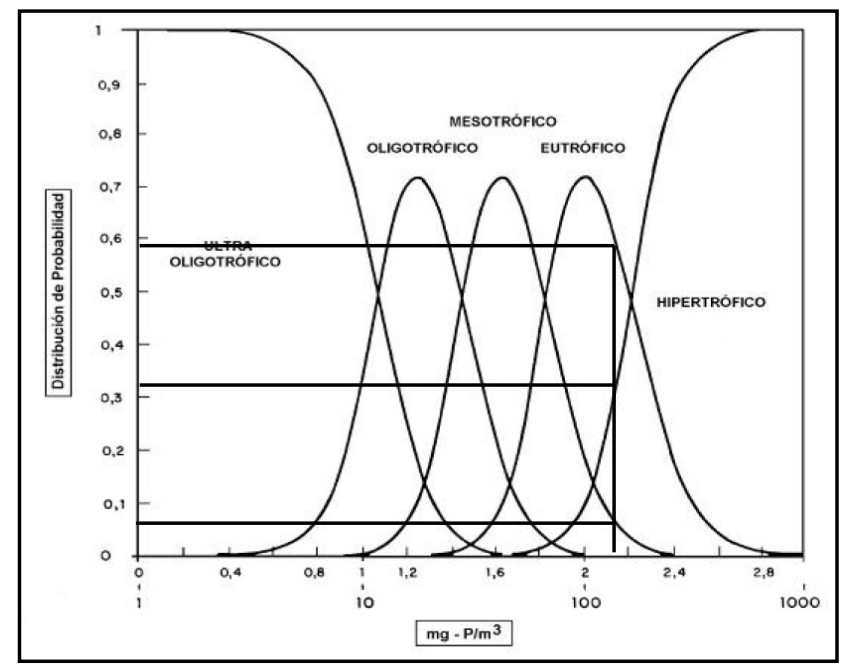

Fig. 4 Distribución de Probabilidad del Estado Trófico Parte Baja Río Tepenaguasapa.

Se pudo comprobar que en los alrededores de donde nace el Río Tepenaguasapa existe una gran actividad ganadera extensiva y con poca vegetación. Siguiendo el curso aguas abajo, en la zona de la parte media del muestreo se encontró con que las concentraciones son similares. Por otra parte, durante el muestreo realizado en la parte baja se produjo un aumento de la probabilidad del estado hipertrófico, aunque, la condición continuó manteniéndose mayoritariamente eutrófica. Esto fue provocado seguramente por el arrastre de los nutrientes de la cuenca, debido al uso que se le da al suelo.

El estado trófico del Río Tepenaguasapa, es consecuencia del inadecuado manejo de la cuenca, lo cual provoca el deterioro del ecosistema. Lo anterior es un resultado de la escasa planificación de las actividades económicas y de asentamiento humano. Esta deficiencia ha permitido la continua expansión de la ganadería extensiva, las áreas dedicadas al monocultivo del arroz, así como también a la falta de control para el tratamiento de las aguas residuales industriales y domésticas. Estas prácticas están causando un proceso de desaparición acelerada del bosque.

La Familia Tubificidae dominó en el río Tepenaguasapa en el mes de enero del año 2001 (Hernández, 2001). Esta Familia es reportada en aguas severamente contaminadas y muy eutrofizadas. También predominaron las cianophytas y eoglenophytas, que igual denotan alto grado de contaminación de agua. 
Asimismo, debe discutirse el efecto biológico de la carga de nutrientes al lago, pues entre sus consecuencias esta la modificación de la estructura de los componentes de la base de la pirámide alimenticia, tal como lo es el fitoplancton.

\section{CONCLUSIONES}

Se encontró un impacto mayor proveniente de la fuente uso de suelo, seguido por el aporte de la precipitación y por último de la carga artificial.

Debido a lo reducido del área de espejo de agua y la gran cantidad de precipitación que en promedio existe dentro de la cuenca, la cantidad de Nitrógeno es mucho mayor que la del Fósforo por la mayor abundancia de este en la atmósfera terrestre.

El sitio en estudio tiene un gran aporte de nutrientes a causa del uso de suelo, debido a que es una zona muy intervenida por la gran cantidad de área utilizada para el cultivo de pastos, tierras dedicadas a la labranza y por que se trata de una cuenca de gran tamaño.

El aporte artificial de nutrientes no es significativo en relación a los aportes de uso de suelo y por precipitación, debido a lo poco poblada que se encuentra la cuenca con 15 habs. $/ \mathrm{km}^{2}$, esto a pesar de no contar con un manejo adecuado de las excretas y las aguas residuales.

El Río Tepenaguasapa posee un gradiente de condición trófica, expresado en $\mathrm{mg} / \mathrm{m}^{3}$, y se caracteriza por presentar una probabilidad mayormente eutrófica cerca del nacimiento del ecosistema hasta un estado que a pesar de ser siempre eutrófico, posee la probabilidad de convertirse en hipertrófico en las cercanías de su desembocadura, en el lago Cocibolca. Todo ello es como resultado de las características ambientales degradadas, reflejadas en una escasa cobertura vegetal (bioindicadores), actividad económica (Ganadería extensiva, monocultivo de arroz y producción láctea), y al asentamiento humano (Carencia de sistemas de tratamiento a las aguas residuales y las excretas), que unidos a la alta precipitación, propician un sustancial transporte de nutrientes, principalmente Fósforo y Nitrógeno.

Los aportes de nutrientes (Fósforo y Nitrógeno) al ecosistema acuático están comprometiendo los usos futuros de las aguas del mismo.
La concentración de nutrientes encontrados en las aguas del ecosistema y el estado trófico del mismo guardan una estrecha relación con la predominancia de los principales grupos fitplanctónicos encontrados en trabajos precedentes, tales como del grupo cianophytas.

La carga de nutrientes de la cuenca de drenaje superficial del Río Tepenaguasapa es significativamente alta, esto es debido al estado eutrófico con tendencia a hipertrófico mostrado en sus aguas.

Uno de los factores que tambien esta aumentando la carga de nutrientes hacia el ecosistema son los incendios forestales, debido a que en Nicaragua esta es una práctica común entre los agricultores (No es una variable incorporada en el método aplicado), esto provoca la pérdida de biomasa, principalmente vegetal y la desnudez del suelo, que facilita a su vez el arrastre de los nutrientes por efecto del viento y/o precipitación. Además, debido a que la cuenca está dominada por amplias zonas con pastizales, que tienden a incendiarse durante la época seca, propiciará que las cenizas aporten el $\mathrm{P}$ y $\mathrm{N}$ de forma más rápida.

Los métodos empleados son aplicables para el tema estudiado, por lo que se sugiere que estas herramientas sean utilizadas en trabajos similares.

\section{REFERENCIAS}

Hernández, S. (2001). Diagnóstico de la Calidad de Agua de los Tributarios que Drenan al Lago Cocibolca. Managua, Nicaragua.

INIDE (2007). Estimaciones y Proyecciones de Población Nacional, Departamental y Municipal: Revisión 2007. Managua, Nicaragua: Instituto Nacional de Información de Desarrollo (INIDE).

INEC (2008). Tabla 3: Población Urbana y Rural de la República, por Departamento y Municipio en los Censos de 1971 y 1995 . Disponible en:

http://www.inec.gob.ni/censo95/censo95.html

JØrgensen, S., \& Vollenweider, R. (1988). Guidelines of Lakes Management: Principles of Lake Management. International Environment Lake Committee. United Nations Environment Programme, 1 91-98. 
Procuenca San Juan. (2004). Diagnostico Ambiental Transfronterizo Procuenca San Juan. Managua, Nicaragua: Publicación Conjunta del Ministerio del Ambiente y los Recursos Naturales de Nicaragua y el Ministerio del Ambiente y Energía de Costa Rica: ARDISA

Salas, H., \& Martino, P. (2001). Metodologías Simplificadas para la Evaluación de Eutroficación en Lagos Cálidos Tropicales. Versión actualizada Enero 2001. Organización Panamericana de la Salud (OPS).

Salas, J. (2002). Biogeografía de Nicaragua. Managua, Nicaragua: Publicación del Instituto Nacional Forestal INAFOR

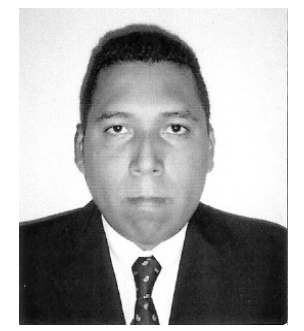

Bismarck Antonio Morales Arróliga graduado de Licenciado en Biología en la Universidad Nacional Autónoma de Nicaragua (UNAN, Managua) en el año 2002. Se graduó en la Maestría en Ciencias Ambientales impartida por el Centro de Investigación y Estudios en Medio Ambiente (CIEMA) de la Universidad Nacional de Ingeniería (UNI) en Managua. Su campo de investigación es la Gestión Ambiental. Actualmente labora para el Ministerio del Ambiente y los Recursos Naturales (MARENA) en el área de recepción y entrega de los permisos y licencias. También se ha desempeñado como profesor de pregrado en la Universidad Central de Nicaragua (UCN), en Managua, Nicaragua.

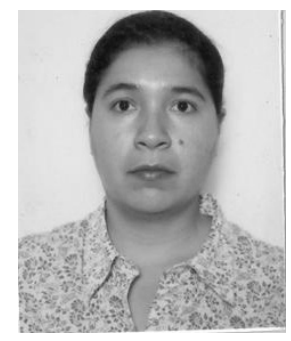

Gabriela Alejandra Chávez Linarte, graduada de Licenciada en Biología en la Universidad Nacional Autónoma de Nicaragua (UNAN, Managua) en el año 2004. Se graduó en la Maestría en Ciencias Ambientales impartida por el Centro de Investigación y Estudios en Medio Ambiente (CIEMA) de la Universidad Nacional de Ingeniería (UNI) en Managua. Su campo de investigación es la Gestión Ambiental. Profesional del Medio Ambiente y actualmente labora en la filial Nicaragua de Cementos Mexicanos (CEMEX) en el área de Medio Ambiente. 